\title{
Transfeminismo y activismos queer: emergencia y cohabitación en las fronteras de la coalición
}

\author{
Transfeminism and Queer Activisms: Emergence \\ and Cohabitation in the Borders of the Coalition ${ }^{1}$
}

PABLO PÉREZ NAVARRO (Centro de Estudios Sociales de la Universidad de Coimbra, Portugal)

Artículo recibido: 7 de noviembre de 2018 Solicitud de revisión: 10 de noviembre de 2018

Artículo aceptado: 8 de enero de 2019

Pérez Navarro, Pablo (2019). Transfeminismo y activismos queer: emergencia y cohabitación en las fronteras de la coalición. Recerca. Revista de Pensament i Análisi, 24(2), pp. 151-172.

\section{Resumen}

El ciclo de protestas que se abrió en las primaveras árabes ha servido para revitalizar la reflexión teórica sobre el modo en que los procesos de agenciamiento político son indisociables de los modos de producción de los espacios de coalición. Es el caso de Judith Butler en Cuerpos aliados y lucha política, junto con otras autoras que han mostrado la importancia de pensar los modos de encuentro corporal, y las políticas de acceso a los espacios de aparición, como parte central de la lucha y la reflexión política. En diálogo con estas y otras provocaciones teóricas, fundamentalmente en el ámbito de los estudios queer, este ensayo explora los modos de producción del espacio de coalición transfeminista y queer a partir de experiencias activistas recientes en la ciudad de Madrid.

Palabras clave: coalición, separatismo, transfeminismo, activismo queer, cohabitación.

Abstract

The cycle of protests opened by the Arab Springs has served to revitalize theoretical reflections on the interplay between the construction of political agency and the production of coalitional spaces. This is the case of Judith Butler in Bodies in Alliance and the Politics of the Street, along with other authors that have shown the importance of thinking bodily encounter, and the politics of accessibility to the space of appearance, as a central part of political reflection and struggle. In dialogue with these and other theoretical provocations, specially

Texto financiado por la Fundação para a Ciência e a Tecnologia no âmbito do Projeto Estratégico do Centro de Estudos Sociais (UID/SOC/50012/2019). 
coming from Queer Studies, this essay explores the modes of production of the space of transfeminist and queer coalition drawing on recent activist experiences in the city of Madrid.

Key Words: coalition, separatism, transfeminism, queer activism, cohabitation.

\begin{abstract}
Mientras que la procedencia designa la cualidad de un instinto, su grado o su debilidad, y la marca que este deja en un cuerpo, la emergencia designa un lugar de enfrentamiento; pero una vez más hay que tener cuidado de no imaginarlo como un campo cerrado en el que se desarrollaría una lucha, un plan en el que los adversarios estarían en igualdad de condiciones; es más bien [...] un no lugar, una pura distancia, el hecho que los adversarios no pertenecen a un mismo espacio. Nadie es pues responsable de una emergencia, nadie puede vanagloriarse; esta se produce siempre en el intersticio.
\end{abstract}

Michel Foucault. Nietzsche, la genealogía, la historia.

\title{
INTRODUCCIÓN
}

El prólogo de Homos, de Leo Bersani (1995), lleva por subtítulo «nosotras»² [we] entre unas prudentes comillas. En él, Bersani manifiesta su extrañamiento ante el modo en que algunas comunidades de pertenencia emergen, incluso, a partir de nuestras formas de tomar distancia de cualquier referente identitario. En otras palabras, a partir de nuestras formas de entrecomillar aquello que hace posible la referencia a cualquier «nosotras». Este reconocimiento deja a Bersani ante un dilema frente a la crítica queer de las políticas de la identidad, por una parte, y con relación a su propia escritura, por la otra, en la que también emergería un «ambiguo nosotras» (Bersani, 1995: 7). Así, en el breve prólogo se celebra y se resiste a un tiempo al modo en que ciertas formas de la crítica queer, desde Monique Wittig hasta Judith Butler, pasando por Michael Warner, reconstruyen una concepción de lo queer que ya no se reconocería tanto en las formas más o menos normativas del deseo como por su forma de distanciarse de los modelos identitarios históricamente construidos en torno a determinadas configuraciones del deseo. En otras palabras, se lamenta Bersani, se habría producido una sublimación de lo queer en posición política que, si

2 Hago uso en esta traducción y en el resto del artículo del femenino universal según el uso del Movimiento 15-M, en el que participé como miembro de la Asamblea Transmaricabollo de Sol. 
bien resulta tan necesaria como irrenunciable, nos deja un cierto sabor a desexualización y a retirada espectral de una comunidad de resistencia que nunca llegó a articularse como tal. Lejos de pretender refugiarse en la nostalgia de la identidad perdida, Bersani lanza la advertencia, propia de una filosofía de la sospecha que no deja de ser, en cierto modo, redobladamente queer, con que se abre este capítulo. La problemática fronteriza de las políticas identitarias tiene una capacidad de replicarse allí donde menos se la espera y también, por supuesto, en el corazón de cualquier disidencia. La alternativa no se encuentra, para Bersani, en la apuesta por algo así como una nueva y más atenta comunidad queer, un tercer socius alternativo, a un tiempo, a la norma heterosexual y a las derivas elitistas, clasistas, racistas, y así ad infinitum, de la comunidad elegetebé. Antes bien, se trataría de impugnar la lógica misma de la construcción comunitaria para pensar otras formas de nosotrear que permitan el encuentro, el cruzamiento y la interferencia entre un imprevisible conjunto de «nosotras». De producirse aún un efecto de comunidad, este sería imposible de fijar por referencia a identidad o preferencia sexual alguna, cuyas formas de pertenencia sean siempre inestables y en la que incluso (como si la blasfemia heterosexual anunciara el éxito del fracaso de la reconstrucción de cualquier nosotras) «muchos heteros puedan encontrar también su lugar» (Bersani, 1995: 22). Se trataría, en suma, concluye Bersani con un notable impulso utópico, de concebir:

Un modo anticomunal de conectar que todas podamos compartir, o un nuevo modo de encontrarnos: esa, y no la asimilación en comunidades previamente constituidas, debería ser la meta de cualquier proyecto para sacar y celebrar al «homo» que todas llevamos dentro (Bersani, 1995: 21).

En los últimos años, la ciudad de Madrid ha sido escenario de una notable proliferación de formas de distanciamientos críticos con los «nosotros» de la sociedad heterocentrada, por una parte, y con la despolitización de las comunidades y luchas elegetebé, por la otra. Estas experiencias nos enfrentan, de lleno, con las ambivalencias a las que se refiere el prólogo de Bersani: así como necesitamos de la apertura del espacio crítico, de la alternativa desde la que articular resistencias que de otro modo permanecerían silenciadas, debemos ser capaces, también, de reconocer los gestos identitarios que compiten por una nueva hegemonía, la reconstrucción de las fronteras que habrían de defendernos de los nuevos otros nosotros. 
La tarea es tanto más urgente dada la proliferación de espacios de protesta producidos, ocupados o transformados por las disidencias sexogenéricas y relacionales, en abierta distancia crítica con el activismo LGTB de signo institucional y el centralismo político y mediático que acompaña, por antonomasia, a la celebración del orgullo LGTB oficial. ${ }^{3}$ Así, por ejemplo, en el 2017, año de celebración del World Pride en Madrid, y en abierta distancia crítica con este, tuvo lugar la celebración del Orgullo de la Periferia, coorganizado entre activistas y colectivos vecinales de hasta nueve barrios de marcado carácter obrero y migrante, con una importante presencia del activismo trans con el lema «No somos MADO, No somos World Pride». ${ }^{4}$ Otros contra-espacios (Foucault, 1967; Lefebvre, 1974) disidentes cuentan con una trayectoria bastante más larga. Este es el caso de la plataforma del Orgullo Crítico, en la que me centraré, que viene funcionando con diferentes nombres desde el año 2006 como estratégico «punto de acumulación» (Redondo, 2015) para una serie de colectivos, por lo general minoritarios, de vida muchas veces efímera y organización asamblearia, interesados en crear alternativas hiperpolitizadas a las celebraciones del orgullo oficial. En este sentido, y a pesar de que la identificación con la palabra queer sea común solo para una parte de los grupos que en ella confluyen, las actividades desarrolladas en torno a la plataforma resultan, como ha señalado Leandro Colling (2015: 199), «fundamentales para entender las diferencias existentes entre el movimiento LGTB y los colectivos queer de España».

Los contra-espacios abiertos por el Orgullo Crítico, como bien recuerda Gracia Trujillo en un artículo reciente (2017), han evolucionado al menos desde el año 2006, cuando grupos reducidos de activistas formaban bloques críticos dentro de la manifestación oficial, hasta la actual manifestación del Orgullo Crítico. Organizada cada 28 de junio desde al menos el 20o8, cuando fue convocada como «Manifestación Orgullo Transmaribollero Anticapitalista» (Artacho, 2008), la manifestación ha crecido de forma exponencial hasta superar las cuatro o cinco mil personas en el 2017 (Ochoa, 2017). El crecimiento ha sido notorio no solo en número de asistentes, sino, sobre todo, de grupos organizados, muchos de ellos emergentes entre los activismos de la disidencia sexogenérica y relacional. Grupos de lesbianas femmes, sexualidades kink y BDSM, asexuales, activistas negras, latinas, el colectivo romaní LGTB, estudian-

3 Me refiero así al conjunto de actividades en torno a la manifestación del Orgullo anual organizadas en colaboración con el Ayuntamiento por un conjunto de empresarios (AEGAL) y colectivos LGTB denominado MADO.

$4 \quad$ Véase: https://www.orgullovallekano.org/ [Consultado el 1 de noviembre de 2018]. 
tes transfeministas y familias de menores trans son, por citar algunos ejemplos, colectivos que han ocupado espacios cada vez más visibles en la manifestación, pronunciando manifiestos a su término, organizando actividades durante los días previos $\mathrm{y}$, en muchos casos, participando además en la asamblea de la plataforma.

En su irreductible pluralidad, el Orgullo Crítico viene funcionando como un buen ejemplo de «heterotopía queer», entendida como la «creación de un espacio donde los individuos fuerzan a la sociedad heteronormativa a reconocer los cuerpos queer como viables en sus propios términos» (Jones, 2009: 6). Al mismo tiempo, en su precaria y agonística unidad, en la plataforma tiene lugar la construcción performativa del sujeto de una lucha colectiva, un nosotrxs que emerge a través de un encuentro que, en palabras de Judith Butler (2017: 77), «configura un espacio que en esencia pertenece a la alianza misma». En relación con los modos de producción de ese espacio de la alianza, podemos convenir con Foucault en que los espacios de la alteridad que bautizara como heterotopías no son, en general, abiertamente accesibles, sino que «o bien la entrada es obligatoria, como en el caso de las barracas o la prisión, bien el individuo se tiene que someter a rituales y purificaciones»o, también, «obtener una cierta autorización, hacer ciertos gestos» (Foucault, 1986: 26). Toda heterotopía dispondría, en este sentido, de una frontera, de permeabilidad variable, que la diferencia de su exterior constitutivo (Mouffe, 1993: 2). El Orgullo Crítico, por ejemplo, acostumbraba a definir sus límites estableciendo las «líneas rojas» que configuraban el horizonte político de la plataforma o, en otros términos, su distancia crítica con aquello contra lo que se moviliza: anticapitalismo, transfeminismo, antiespecismo o antirracismo son, por ejemplo, algunas de esas líneas que varían en función del consenso anual.

En lo que sigue, en la línea de las ambivalencias entre comunidad y conexión o, como sugeriré más tarde siguiendo a Samuel R. Delany, entre comunidad y contacto, pretendo abordar críticamente los efectos de la política de fronteras adoptada por la plataforma al definirse como, usando una fórmula de larga tradición separatista, un espacio «no mixto» (Orgullo Crítico, 2017). A pesar de limitar el análisis, aquí, a las políticas espaciales del entorno del Orgullo Crítico de Madrid, las dinámicas entre separatismo y cohabitación apuntan a desafíos fundamentales para las políticas de alianzas en un sentido amplio. Existe, en este sentido, un brusco hiato entre la adopción del separatismo por parte de colectivos o incluso bloques concretos - por sí misma, una fuente inagotable de conflictos de frontera, como recuerda Ricky G. Robles en «Repensando los bloques no mixtos» (2018) - y su extensión a los espacios de 
confluencia y coalición. Considerando esta transición, cabe preguntarse ¿qué efectos tiene la producción separatista del espacio de coalición sobre la articulación del sujeto de la lucha colectiva? ¿Sobre qué exclusiones constitutivas descansa la coalición no mixta y en qué medida su topología resulta de o, más bien, precede a la apertura del espacio de encuentro? ¿Cómo se relacionan temporalidad y legitimidad en la adopción del separatismo como modelo de cohabitación? ¿Qué formas de encuentro resultan legitimadas y cuáles relegadas a los márgenes de la emergente alianza? ¿Qué convergencias entre luchas se ven así reforzadas y cuáles condenadas, en fin, al ostracismo de la alianza ininteligible?

\section{ESPACIALIDADES ABIERTAS, SECURITARIAS E IDENTITARIAS}

Llevando a una suerte de límite la inclusividad de un separatismo que dista de ser reducible al de los espacios women only del feminismo radical, la asamblea organizativa del Orgullo Crítico pasó a definirse, en el 2017, como un espacio «no mixto» para personas «que se sienten parte de alguna disidencia sexual y de género» (Orgullo Crítico, 2017). Para entender mejor la especificidad de esta fórmula y el lugar que ocupa en relación al conjunto butleriano de

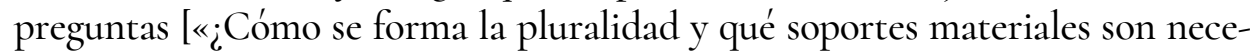
sarios para su formación? ¿Quién entra en esta pluralidad y quién no? ¿Y cómo se decide eso?» (Butler, 2017: 82)], resulta útil explorar, así sea brevemente, las diferentes políticas espaciales características de diferentes grupos del entorno activista de la plataforma.

Un cierto extremo es el que representa la Asamblea Transmaricabollo de Sol. Fiel a los principios del antiausteritario Movimiento 15-M, al que pertenece (Pérez Navarro, 2015), sus asambleas son abiertas, convocadas públicamente, sin cualquier tipo de limitación sobre el conjunto de géneros, sexualidades o (des)identificaciones invitadas para proponer o bloquear consensos en cada asamblea. Claro está, la vocación de apertura radical de esta asamblea en particular, o la del 15-M en general, no debe ser confundida con una suerte de producción voluntarista de un espacio donde las diferencias de género, clase, raza, diversidad funcional o cualesquiera otras no puedan operar como políticas de frontera de formas más o menos implícitas. De hecho, grupos como Feminismos Sol y la propia asamblea desarrollaron una importante labor crítica frente al sexismo, la homofobia y la transfobia, entre otras fuerzas exclu- 
yentes, en la acampada inaugural del movimiento (Pérez Navarro, 2014): existe siempre una distancia entre la pretendida asepsia inclusiva de la utopía y su siempre ambivalente concreción heterotópica.

Más explícitamente delimitados se encuentran los espacios definidos como seguros, siguiendo una denominación ligada a la historia de los espacios autónomos del feminismo radical en los años 70 (Kenny, 2001: 24). De forma más reciente, la expresión espacios seguros remite también a la práctica de habilitar espacios libres de violencias homofóbicas o transfóbicas, entre otras, especialmente en el ámbito académico estadounidense. Cabe señalar que, lejos de suscitar consenso como estrategia en el ámbito, algunas teóricas queer encuentran una fuerte connotación neoliberal en el «foco en la seguridad y la vigilancia» (Quinan, 2016: 362) en este énfasis securitario, así como una problemática fobia al conflicto (Halberstam, 2014).

En el caso concreto del activismo de la ciudad de Madrid, es muy común el uso de la expresión en múltiples centros sociales ocupados autónomos, así como en multitud de fiestas, encuentros y asambleas del entorno de la disidencia sexogenérica y relacional. Por ejemplo, un colectivo de formación reciente, Sección Invertida, define sus espacios como seguros en el sentido de que en ellos las actitudes machistas, homofóbicas o discriminatorias de cualquier tipo no van a ser toleradas. 5 Por lo que a la política de fronteras se refiere, cabe señalar que este recurso a la noción de seguridad no determina por anticipado quién puede sumarse o no al espacio común. No obstante, la espacialidad securitaria restringe la permeabilidad del encuentro al anunciar la potencial expulsión de quien entre en conflicto con los consensos, explícitos o no, que rigen sobre los modos de cohabitación.

Por último, muchas veces, en combinación con la anterior apuesta por los espacios seguros, existe también la posibilidad de delimitar los exteriores constitutivos de las asambleas en términos identitarios, como hace una parte importante de los colectivos definidos como transfeministas. Término este que, dada la diversidad de tradiciones activistas y de políticas espaciales a la que apunta, precisa de una atención específica.

\footnotetext{
Como explican en su manifiesto. Véase: https://seccioninvertida.wordpress.com/manifiesto/.
} 


\section{DECLINACIONES DEL TRANSFEMINISMO}

Los transfeminismos cuentan con una amplia diversidad de genealogías posibles. No obstante, en el caso concreto del Estado español sería difícil no incluir entre estas dos influencias claras: el movimiento por la despatologización de las identidades trans ${ }^{6}$ y el impacto de los activismos queer en diferentes espacios del feminismo autónomo (Fernández y Araneta, 2013). Como resultado de esta convergencia, uno de los rasgos definitorios del transfeminismo será la apertura crítica de las políticas feministas del sujeto único en dirección a una alianza entre una multiplicidad de corporalidades, géneros y posicionamientos subjetivos. En ese sentido, el transfeminismo participa de las dinámicas entre proliferación y coalición intrínsecas a las políticas queer desde los tiempos de la respuesta activista a la crisis del sida y que se reflejan, en el ámbito de la teoría, en obras como El género en disputa (Butler, 2007).

Este impulso proliferante se encuentra presente de forma especialmente rotunda en el «Manifiesto para la insurrección transfeminista» de la Red PutaBolloNegraTransFeminista (RED, 2009). Firmado por un conjunto de colectivos identificados como «manada furiosa», este referente fundacional del transfeminismo acoge una serie amplia de posicionamientos y (des)identificaciones que desborda cualquier marco de referencia común posible. Putas, bollos, sin papeles, negras, precarias, trans, maricas, musulmanas, travestis y heterodisidentes, entre otras, se dan cita sin orden aparente en el manifiesto de la Red. Más que una coalición concreta entre una serie de identidades, compuesta, entre otras, por «hablantes de wolof», la Red anunciaba así su apuesta por una política de la emergencia, constituida por una serie incoherente de alianzas impredecibles inscrita en la desorganización taxonómica del manifiesto y su recurrente uso de los puntos suspensivos.

Esta irrupción transfeminista [término que, para algunas, sonaría «mejor en castellano que el término queer» (Solá, 2013: 19)] ha evolucionado por diferentes caminos. En la actualidad, una parte importante de los colectivos transfeministas apuesta por combinar el impulso proliferante, reconociblemente queer, inscrito en el manifiesto, con la inercia de las vertientes separatistas de parte del feminismo radical («Venimos del feminismo radical», comenzaba el

Articulado, fundamentalmente, en torno a la campaña internacional Stop Trans Pathologization. Véase: http://stp2012.info/old/es [Consultado el 1 de noviembre de 2018]. 
manifiesto de la Red). Así, existen múltiples espacios que desbordan las políticas del sujeto único mujeres (y, en ese sentido, son transfeministas) delimitados por fronteras bien definidas en términos identitarios (y, en ese sentido, son separatistas). Un buen ejemplo lo podemos encontrar en algunos de los centros sociales emblemáticos del activismo feminista de Madrid, como la Eskalera Karakola, activa desde 1996. Este espacio autónomo se define como transfeminista ${ }^{7}$ y tanto sus asambleas organizativas como parte de sus actividades son «no mixtas» en el sentido de estar reservadas, en concreto, a la participación de «mujeres, bolleras y trans». ${ }^{8}$ De forma similar, otro espacio social autónomo, la Hoguera Transfeminista, desalojado en el 2015 pero que mantuvo su continuidad como colectivo, se definía como un espacio ocupado también por mujeres, bolleras y trans en una «apuesta clara por lo que llamamos 'espacios no mixtos' o 'espacios diferenciados', donde las masculinidades cis y la heterosexualidad dejen de tener el espacio que ocupan en nuestro sistema hetero-patriarcal-capitalista» (La Hoguera Transfeminista, 2014). No obstante, la Hoguera explicitaba su interés por ampliar de forma puntual los límites de sus espacios diferenciados hasta «generar algunos lugares» donde los «colectivos transmaricabollo, que incluyen hombres maricas, puedan sentirse confortables».

Otro colectivo que se presenta como transfeminista y que apuesta por el separatismo de los espacios no mixtos es Migrantes Transgresorxs, cuyo activismo resulta de un intenso cruce entre las luchas de las «personas racializadas, migrantes, negrxs, refugiadxs diversxs»` y las disidencias sexuales y de género. Especialmente a partir del 2017, el grupo ha sido muy activo en la producción de espacios no mixtos accesibles, en este caso, «solo para personas racializadas», en la forma de encuentros y talles sobre antirracismo, gordofobia o antiespecismo, entre otros temas, además de alguna fiesta expresamente anunciada como mixta. En el marco del Orgullo Crítico, Migrantes participó de la convocatoria de un bloque no mixto para la manifestación, conformado por «solo racializadxs, migrantes, negrxs, afrodiasporicxs, sudakxs, morxs, gitanxs, asiáticxs, árabes y rumanxs» (Migrantes Transgresorxs, 2017) y presentado como Manada Antirracista (Trans)fronteriza y «libre de euroblancos». Formulación

Véase: https://eskalerakarakola.org/proyectos-karakola/ [Consultado el 1 de noviembre de 2018].

8 Tales como sus asambleas organizativas internas o la denominada «Asamblea abierta de Lavapiés. No mixta» de preparación de la huelga feminista del 8 de marzo (https://www.facebook.com/events/382691368810028/; cursivas propias) [Consultado el 1 de noviembre de 2018].

9 Véase: http://migrantestransgresorxs.blogspot.pt/p/quienes-somos.html [Consultado el 1 de noviembre de 2018]. 
esta última que, con gran capacidad sintética, combina la apuesta por un separatismo declinado en términos raciales con la referencia a una proliferación que se transmuta, en el mismo gesto, en apropiación de la injuria de la animalización colonial, denunciada entre otros por Frantz Fanon, del cuerpo racializado. La preocupación de Migrantes por «deconstruir y construir espacios» no solo ocupa una parte central en su discurso, sino que se extiende a los tránsitos de género a través de la denuncia de las violencias que recaen sobre quien cruza, a un tiempo, las fronteras entre «geografías y territorios corporales». ${ }^{10}$

A las declinaciones del transfeminismo pertenecen también encuentros estatales como el celebrado en Madrid en el año 2015 y definido como «transfeminista, libertario, no mixto» (Encuentro Marica, 2015), que se dirigía a «personas que se identifiquen con la etiqueta marica». La multiplicidad transfeminista se encontraba nuevamente presente en una proliferación interna a la identidad marica, para incluir un «amplio abanico de identidades existentes, personas trans, identidades de género no binario... que sientan como suyo ese preciado término». El encuentro se proponía superar el olvido, entre la multiplicidad de luchas, de aquello «que nos compete específicamente como maricas» (2015, cursivas mías) y sirvió de base para la formación de otras asambleas maricas y no mixtas que también tuvieron presencia en el Orgullo Crítico. ${ }^{11}$ Los encuentros estatales, a su vez, también han tenido continuidad. Así, el del 2017 tuvo lugar en Granada y planteaba, como Migrantes, una geografía política a un tiempo territorial y sexogenérica, con el fin de «articular disidencias y resistencias y construir alternativas desde nuestra diversidad de identidades marikonas vivenciadas desde el sur»(Encuentro Marica, 2017). Esta referencia al sur se presentaba, en la convocatoria, como «territorio geográfico pero también político y simbólico atravesado por diferentes relaciones de poder, (des)prestigios, (des)privilegios y opresiones». ${ }^{12}$ De este modo, la política de la no mixticidad marica combinaba el sacrilegio de un transfeminismo sin mujeres, la proliferación interna a un único marco identitario y una apropiación desviada de las epistemologías del sur de Boaventura de Sousa Santos.

En su diversidad, este conjunto de políticas espaciales comparte la tentativa de definir su «régimen particular de aparición» (Butler, 2017: 82) en los términos provistos por diferentes series de referentes identitarios. En este

10 Véase: http://migrantestransgresorxs.blogspot.pt/p/quienes-somos.html [Consultado el 1 de noviembre de 2018].

11 En el año 2016, por ejemplo, estas incluían la celebración de una fiesta «no mixta de corporalidades diversas, abierta a todas las personas que se identifiquen con la etiqueta política 'Marika'».

12 Ídem. 
sentido, las declinaciones separatistas del transfeminismo contrastan vivamente con políticas de alianzas como las inscritas en el manifiesto de la Red, sin duda más próximo a la noción de multitudes queer con que uno de sus signatarios destacados, Paul B. Preciado, resignificaba el indelimitable sujeto de la revolución articulado por Hardt y Negri (Preciado, 2003: 2). Por otro lado, en los términos de una topología de las alianzas, esta peculiar dinámica entre impulsos proliferantes y contenciones identitarias encuentra un interesante paralelismo en la tensión que existe entre el prólogo y la introducción de Transfeminismos. Epistemes, fricciones y flujos (VV. AA., 2013), una antología que también ha funcionado como referencia para el transfeminismo en el Estado español. Así, en «Decimos revolución», Paul B. Preciado afirma el efecto desestabilizador de una indelimitable y proliferante «diáspora rabiosa» (Preciado, 2013: 12) sobre las dicotomías «hombre/mujer, blanco/negro, humano/animal, homosexual/heterosexual, válido/inválido, sano/enfermo, loco/cuerdo, judío/musulmán, Israel/Palestina» (Preciado, 2013: 11). En abierto contraste, el texto de Miriam Solá que le sigue celebra el distanciamiento de los transfeminismos respecto al carácter más confrontacional de lo queer. De suerte que, tan solo cuatro años después de que la citada Red denunciara en las Jornadas Feministas Estatales de Granada del 2009 el carácter excluyente de un sujeto mujer que «se nos había quedado pequeño» (RED, 2009), el movimiento habría madurado en una dirección que se apunta como más responsable y más «humilde» (Solá, 2013: 19) hasta el punto de dejar de gritar que «el feminismo será transfeminista o no será» (Solá, 2013: 22, n. 9) porque, por fortuna, siempre según Solá, al feminismo «no está en nuestra mano cambiarlo» (Solá, 2013: 22).

Así descrita, esta conciliadora evolución ${ }^{13}$ y la propia tensión interna entre proliferación y contención identitaria de los separatismos transfeministas adquiere los rasgos de la conocida secuenciación con que Jeffrey Weeks pretendía domesticar el potencial crítico del momento del rupturismo y la transgresión queer a una mera fase preparatoria de la supuestamente más madura etapa de la negociación de demandas (Weeks, 1995: 12). Con la particularidad, eso sí, de que esta contención separatista se encontraría aquí, al mismo tiempo, antes y después del impacto de la crítica queer sobre los espacios del feminismo autónomo.

13 De la que no participan muchos colectivos transfeministas y transmaricabollos, como puede reconocerse, por ejemplo, en el uso del mismo lema «El feminismo será transfeminista o no será» por la Asamblea Transmaricabollo de Sol en la manifestación del 8 de marzo de 2012 o el aún más confrontacional «Las bolleras no somos mujeres» en el bloque no mixto de la misma manifestación, ya en el 2018 (Asamblea Transmaricabollo de Sol, 2018). 


\section{EL ESPACIO DE LA COALICIÓN}

A la hora de pensar las políticas espaciales necesarias para el encuentro entre colectivos en espacios de coalición como el que representa la plataforma, se puede señalar, en primer lugar, que, en un sentido estrictamente formal, los diferentes separatismos acostumbran a ser incompatibles entre sí. El separatismo de espacios como la Hoguera no cabe en los separatismos migrantes que, a su vez, no caben en espacios no mixtos monoidentitarios como el del separatismo marica. La posibilidad de una cohabitación entre los mismos, entendida esta como una «coarticulación con la alteridad», como lo expresa Butler (2012: 3o), dependerá por tanto de la adopción de una política espacial diferente a la de cualquiera de ellos. De forma más brusca, si cabe, las (des)identificaciones posibles en asambleas abiertas, como las de diversos colectivos que participan en el Orgullo Crítico, desborda los límites de cualquier marco separatista. Así, el carácter no mixto de la asamblea consensuado en el 2017 excluyó de la participación, por ejemplo, a cualesquiera aliadas de la disidencia sexual o de género, a pesar de que estas pudieran de hecho participar en muchos de los grupos que componen la plataforma. Al mismo tiempo, esta no mixticidad del espacio de confluencia erige una «frontera discursiva» (Butler, 2017: 5) ante quienes entienden que sus disidencias se encuentran más bien yuxtapuestas, pero no subsumidas, en la sexual o de género. El efecto contrario no se verifica: en espacialidades abiertas como la que venía representando la plataforma del Orgullo Crítico caben tantos colectivos separatistas como se quieran.

En una perspectiva más situada, cualquier consenso alcanzado sobre la composición mixta o no mixta implica un diferencial de legitimidades que se distribuye sobre un eje que no es tan solo espacial, sino también temporal. Por definición, las políticas de la no mixticidad resultan de un momento de clausura en el que una serie de participantes establece la política de frontera que se debe aplicar sobre quienes puedan sumarse posteriormente. De esta forma, la temporalidad del separatismo inscribe, en los modos de producción del espacio de la coalición, las dinámicas éticas y políticas propias de las jerarquizadas relaciones entre el anfitrión y el huésped. Y lo hace en un sentido que no es solo sincrónico sino, a través de todos los sobreentendidos implícitos en cualquier definición del carácter no mixto, también histórico. Esta clausura interna del proyecto separatista se define, entre otras cosas, por su renuncia a encarar el desafío de una «dificultad de la traducción» que Judith Butler relaciona en los siguientes términos con el problema general de la temporalidad (Butler, 2012: 130, traducción propia): 
El co de la cohabitación es también un nexo donde temporalidades convergentes articulan el momento presente, no un momento en el que una historia de sufrimiento niegue a la otra, sino en el que se hace posible que una historia de sufrimiento aporte las condiciones de armonización con la otra, y donde cualesquiera que sean los vínculos establecidos procedan a través de la dificultad de la traducción.

De forma similar al modo en que ha operado la transfobia en los espacios no mixtos del separatismo lesbiano, ${ }^{14}$ los colectivos y discursos de incorporación más reciente parecen ser los que con mayor facilidad sufren las dificultades de estos ejercicios de traducción cuya inevitabilidad resulta, por parafrasear la idea de Arendt que retoma Butler con frecuencia (véase, por ejemplo, Butler, 2014), del hecho de que no elegimos, al menos no a priori, con quién cohabitamos en los espacios de coalición. Tal parece haber sido el caso, por lo que se desprende del manifiesto que leyeron en el punto final de la manifestación del Orgullo Crítico, del colectivo asexual ACEs (2017): $:^{15}$

Podéis echarnos de todos los espacios que queráis, pero la calle es nuestra. Nuestra presencia en espacios transfeministas es muchas veces leída como una intromisión, como una molestia, como un cuestionamiento de las libertades sexuales. Libertad sexual también es no follar, follar solo con personas a las que queremos, follar poco, deconstruir el deseo. Venimos a decir que lo transfeminista puede entenderse desde otros escenarios que no mitifiquen el sexo. [...] Hemos forjado alianzas inesperadas con otras disidencias. Formamos redes más diversas, más visibles. Ojalá sea la base de otro tipo de orgullo. Otra forma de compartir espacios.

Este colectivo asexual reflejaba así, por una parte, una distancia crítica similar a la que describe Gavin Brown (2007) en referencia a espacios autónomos del activismo queer de la ciudad de Londres. En la ocasión referida por este geógrafo de las espacialidades queer, un grupo de activistas irónicamente autodenominados como Alianza Frígida Juvenil llamó la atención sobre la incomodidad que la sexualización del espacio podía tener sobre «éticas sexuales diferentes» (Brown, 2007: 2695). La Alianza Frígida habría venido en aque1la ocasión a recordar, apunta Brown, que la asexualidad también puede servir para queerificar los espacios queer (Brown, 2007: 2695). Por el otro, la intervención de ACEs dejaba constancia de una «expulsión» que trasciende los límites

14 Como el que acompañó los 40 años de vida del Michigan Womyn's Music Festival, mítico enclave del separatismo lesbiano.

15 Véase: https://www.asexual.es/ [Consultado el 1 de noviembre de 2018]. 
de la mera divergencia crítica. ${ }^{16}$ En su aspecto más elemental, esta ruptura marca el fracaso en el proceso de convertir conflictos y antagonismos en un agonismo, por usar aquí los términos con que Chantal Mouffe (2016) describe el objetivo de cualquier política democrática, que no interrumpa la convivencia política. Al mismo tiempo, la referencia a la «expulsión», ya sea esta metafórica o literal, expone claramente la distribución desigual de legitimidades intrínseca a la temporalidad del separatismo en los espacios de coalición. Lejos de limitarse en mi opinión a conflictos concretos, de mayor o menor carácter anecdótico, estas dinámicas de la (in)hospitalidad se extienden, en potencia, a los colectivos que habitan en las fronteras temporales de la disidencia sexogenérica y relacional: activismos poliamorosos y no monógamos, colectivos que son, por definición, de aliadas, como las familias de menores trans, los emergentes activismos de las comunidades kink y BDSM o los y las trabajadoras del sexo, por citar solo algunas. Se extienden, especialmente, cuando cuentan entre sus filas, como todos los anteriores, con sujetos situados en el exterior constitutivo por antonomasia de los separatismos feministas, incluyendo al transfeminista: el lugar que representan los activistas identificados o leídos como hombres cis y heterosexuales.

En cualquier caso, la producción separatista de los espacios de coalición no solo anuncia potenciales conflictos fronterizos, como si de los orgullos críticos con el Orgullo Crítico se tratara; también delimita, como gesto definitorio, los que ni siquiera van a poder ser planteados. Parafraseando las topologías de la inteligibilidad descritas por Judith Butler en «Is Kinship Always Already Heterosexual?» (2002), tales conflictos apuntarían a coaliciones que resultan ininteligibles para el proyecto separatista. Entre estas se encuentran, paradójicamente, algunas de las alianzas a las que apuntan los debates que más fuerza han cobrado en la plataforma de forma reciente. Este es el caso, por ejemplo, de la crítica a la construcción homonacionalista (Puar, 2017) de las políticas LGTB a través de la construcción de, entre otras, la figura del refugiado islámico como amenaza homofóbica (Ahmed, 2017). Pertenecen igualmente a este espacio de la alianza ininteligible una multitud de luchas que intersectan con cualquier concepción posible de la disidencia sexogenérica y relacional, como el antifascismo, las movilizaciones contra la represión de la protesta y los ataques a la libertad de expresión, las luchas vecinales contra la gentrificación de

16 La situación recuerda a la que se han enfrentado otros colectivos emergentes en los espacios de la disidencia sexogenérica y relacional, como la tentativa de expulsión de las activistas del poliamor Poliportugal del Orgullo LGTBQ de Lisboa (Cardoso, 2014). 
Lavapiés, barrio en que habita el Orgullo Crítico, o las cada vez más intensas luchas de la población migrante (sin énfasis en la cuestión sexogenérica), por citar solo algunas de las alianzas con las que el proyecto separatista renuncia a cohabitar en su propio espacio y, con ello, a perderse en el no lugar —en el sentido en que Foucault usa esta expresión en la cita que abre este ensayo- de una impredecible emergencia.

\section{PUENTES, CONTACTOS Y FRONTERAS: A MODO DE CONCLUSIÓN}

En el par de ensayos que componen Times Square Red, Times Square Blue, Samuel R. Delany (2001) expone el mundo de relaciones que los teatros X y otros locales similares hacían posible en la Nueva York de los años 8o. Relaciones no solo sexuales, sino también de amistad, establecidas entre un amplio espectro de diferencias raciales, sexuales, de clase, generacionales o de diversidad funcional. La pérdida de la riqueza política, individual y, sobre todo, intercomunitaria que la reestructuración neoliberal y conservadora del espacio urbano representó queda identificada, en su relato, con la destrucción de estas pornotopías (Preciado, 2010) en que se intensificaban las ocasiones para lo que Delany denomina contacto: el tipo de encuentro del cual surgen alianzas sexuales, amistosas, puntuales o duraderas, entre personas muy diferentes entre sí. La posibilidad de este tipo de contactos depende, aprendemos de Delany, de la disponibilidad de espacios cuya alteridad radica en su modo de hacer posible el encuentro en y desde la diferencia: «El contacto urbano suele alcanzar su máximo beneficio cuando ocurre entre miembros de diferentes comunidades. Por eso mantengo que el contacto interclase es aún más importante que el intercambio intraclase» (Delany, 2001: 127). En este sentido, la diferenciación y segregación de los espacios en la ciudad neoliberal (Alves, 2014: 2) contra los que nos prevenía, entre otros, Henri Lefebvre representaría un ataque directo a las posibilidades para establecer los contactos de los que dependemos para la multiplicación de las alianzas y la intersección de las resistencias.

El aspecto sexual implícito en la metáfora de Delany alude a la posibilidad de una desaparición de la distancia entre superficies corporales no obstante diferenciadas de la que depende, acaso, el carácter político de cualquier encuentro. Pensar la producción del espacio de coalición a partir de esta idea pasaría por asumir los riesgos de una cohabitación radical entendida como posibilidad de colapso de ese espacio político entre los cuerpos que, para Butler, «une tanto como diferencia» (2017: 77). La apertura del (a)sexualizado 
espacio de la coalición dependería así de las ambivalencias topológicas de una separación que es, como la dificultad de la traducción, al mismo tiempo frontera y puente. ${ }^{17}$ Tal es la política espacial que define, en mi opinión, los modos históricos de producción del espacio queer, y que Gloria Anzaldúa (2002: 2) denominaba, simplemente, puentear:

No hay espacios seguros. El «hogar» puede ser inseguro y peligroso porque implica la apariencia de la intimidad y, con ella, fronteras más finas. Permanecer en el «hogar», sin tomar el riesgo de salir de nuestro propio grupo, es una actitud que procede de la herida, y estanca nuestro crecimiento. Puentear significa debilitar nuestras fronteras, no cerrarlas a los otros. Puentear es el trabajo de abrir la puerta al extraño, por dentro y por fuera. Cruzar el umbral es deshacernos de la ilusión de seguridad porque nos sitúa en un territorio que no es familiar y que no garantiza la seguridad. Puentear es una tentativa de comunidad, y para eso debemos tomar el riesgo de estar abiertos a la intimidad personal, política y espiritual, tomar el riesgo de ser heridas.

Los espacios del activismo tienen sus lógicas situadas, sus dinámicas y contradicciones, incluyendo momentos de mayor o menor apertura a establecer alianzas con otros movimientos. Resulta importante pensar, no obstante, los modos en que las políticas separatistas pueden frenar alianzas frente a desafíos que, como la higienización moral y securitaria del espacio público o la connivencia entre las luchas LGTB y la derecha xenófoba, afectan a una multiplicidad de sujetos imposible de contener en ningún espacio diferenciado. La posibilidad de la disidencia, de la disidencia como posibilidad, depende acaso de nuestra capacidad para multiplicar puentes y superficies de contacto, para transformar cualquier coalición en aquello que esta todavía no es y, en suma, para habitar unas políticas comprometidas con su propio desbordamiento crítico. Estos desbordamientos pueden contemplarse como perturbaciones internas, como cuando Butler se refería, al comienzo de El género en disputa, a la genealogía feminista de la crítica queer en los términos de una «división en el seno del feminismo y la oposición paradójica a él por parte de las mujeres a quienes dice representar» (Butler, 2007: 51, cursivas mías). Desde otro punto de vista, el mismo tipo de conflicto se puede leer más bien como una perturbación o un proceso de emergencia que tiene lugar en o desde los márgenes del espacio de coalición.

17 A esta ambivalencia entre puentes y fronteras se refería también Boaventura de Sousa Santos en un artículo reciente: «As fronteiras entre muros e travessias» (2018: 27). 
En el caso del Orgullo Crítico, estas perturbaciones se pueden asociar con lo que podríamos entender como bloques críticos de o con, según el caso, la propia plataforma, que tienen ya una intermitente historia y que parecen más frecuentes al tiempo que esta aumenta su complejidad organizativa. Una de sus manifestaciones más recientes tuvo lugar, sin ir más lejos, durante el Orgullo Crítico del 2018, mientras reviso las conclusiones de este artículo, en la forma de una crítica a la organización por su estricto respeto a los horarios de manifestación previamente convenidos con la policía y, en particular, a las indicaciones de esta in situ. Esto habría impedido que se leyera, durante el micro abierto final, el manifiesto del bloque de migrantes y racializadas. Estas no tardaron en denunciar que justamente ellas tendrían, por sus situaciones de irregularidad administrativa, más motivos para temer a la policía que la mayoritariamente blanca organización del Orgullo Crítico:

Por ingenuidad, por inocencia blanca o por alguna otra razón, la jerarquía racial segregacionista del estado racista español se remarcó al final de «la celebración del Pride». Somos los cuerpos a los cuales la policía persigue, encierra, deporta y asesina. Amigx blancx, la policía no viene por ti, viene a por nosotrxs (Bloque Antirracista, 2018).

El posterior comunicado de respuesta a este desencuentro (Orgullo Crítico, 2018) llama la atención, entre otras cosas, ${ }^{18}$ por su elevado nivel de autocrítica, constatando que estas tensiones y conflictos en los márgenes no tienen por qué constituir una fuerza centrípeta. Antes bien, anuncian posibilidades de transformación crítica inscritas en las fronteras de cualquier espacio identitario, cualquier comunidad, cualquier alianza de cuerpos en resistencia.

\section{BIBLIOGRAFÍA}

ACEs, Asexual Community España (2017). Manifiesto Asexual en el Orgullo Crítico Madrid [Orgullos Críticos do Sul]. Recuperado de: https://orgulloscriticos.wordpress.com/2017/o7/o3/manifiestoasexual-en-el-orgullo-critico-madrid/. [Consultado el 1 de noviembre de 2018].

Ahmed, Daniel (2017). Islamofobia queerizada e islam queer en tiempos

18 Pero también por el llamamiento final a hacer «un orgullo crítico más seguro para todas», en especial teniendo en cuenta que el silenciamiento se habría originado justamente al poner el ideal de la seguridad por encima de otras variables. 
de homonacionalismo. En SOS Racismo (Org.). Islamofobia y género. Mujeres, feminismos, instituciones y discursos frente al sexismo y el racismo (61-64), SOS Racismo.

Alves dos Santos Junior, Orlando (2014). Urban Common Space, Heterotopia and the Right to the City: Reflections on the Ideas of Henri Lefebvre and David Harvey. Brazilian Journal of Urban Management, 6(2), 146-157. doi: 10.7213/urbe.06.002.SEo2.

Anzaldúa, Gloria (2002). (Un)natural Bridges (Un)safe Spaces. En Anzaldúa, Gloria y Keating, AnaLouise (Eds.). This Bridge We Call Home. Radical Visions for Transformation (1-6). Abingdon: Routledge.

Artacho, Francisco (25 de junio, 2008). Orgullo Anticapitalista Rosa. [El becario en bici]. Recuperado de: http://elbecarioenbici.blogspot.pt/2008/o6/orgullo-anticapitalistarosa.html [Consultado el 1 de noviembre de 2018].

Asamblea Transmaricabollo de Sol (8 de marzo de 2018). Manifiesto Bollotransputa para el 8M18. [Transmaricabollo de Sol]. Recuperado http://asambleatransmaricabollodesol.blogspot.pt/2018/o3/manifies to-bollotransputa-para-el-8m18.html [Consultado el 1 de noviembre de 2018].

Bersani, Leo (1995). Homos. Cambridge/London: Harvard University Press.

Bloque Antirracista (28 de junio, 2018). El silenciamiento blanco es una estrategia histórica para opacar nuestras voces [Negrxs]. Recuperado de: https://www.negrxs.com/new-blog/2018/6/29/elsilenciamiento-blanco-es-una-estrategia-histrica-de-opacarnuestras-voces [Consultado el 1 de noviembre de 2018].

Brown, Gavin (2007). Mutinous Eruptions: Autonomous Spaces of Radical Queer Activism. Environment and Planning A: Economy and Space, 39(11), 2685-2698. doi: 10.1068/a38385.

Butler, Judith (2002). Is Kinship Always Already Heterosexual? Differences: A Journal of Feminist Cultural Studies, 13(1), 14-44. doi: 10.1215/10407391-13-1-14.

Butler, Judith (2007). El género en disputa. El feminismo y la subversión de la identidad. Barcelona: Paidós.

Butler, Judith (2012). Parting Ways. Jewishness and the Critique of Zionism. New York: Columbia University Press. 
Butler, Judith (2014). Willing the impossible: an interview with Judith Butler. Transformation. Where love meets social justice Recuperado de: https://www.opendemocracy.net/transformation/rayfilar/willing-impossible-interview-with-judith-butler [Consultado el 1 de noviembre de 2018].

Butler, Judith (2017). Cuerpos aliados y lucha política: hacia una teoría performativa de la asamblea. Madrid: Paidós.

Cardoso, Daniel (2014). My Spivak Is Bigger Than Yours: (Mis)Representations of Polyamory In The Portuguese LGBT Movement And Mononormative Rhetorics. LES Online, 6(1), 45-64.

Colling, Leandro (2015). Que os outros sejam o normal. Bahia: Edfuba.

De Sousa Santos, Boaventura (2018). As fronteiras entre muros e travessias. Jornal de Letras, Artes e Ideias, 1237, 27.

Delany, Samuel R. (2001). Times Square Red, Times Square Blue. New York: New York University Press.

Encuentro Marica (2015). Convocatoria para un Encuentro Marika transfeminista libertario estatal [Encuentro Marica]. Recuperado de: https://encuentromarika.noblogs.org/post/2015/11/ [Consultado el 1 de noviembre de 2018].

Encuentro Marica (2017). Encuentro «Ponme mirando al sur, marikón» [Encuentro Marica]. Recuperado de: https://encuentromarika.noblogs.org/post/2017/o3/14/encuentroponme-mirando-al-sur-marikon/ [Consultado el 1 de noviembre de 2018].

Fernández, Sandra y Araneta, Aitzole (2013). Genealogías Trans(feministas). En Solá, Miriam y Urko, Elena (Comp.). Transfeminismos. Epistemes, fricciones y flujos (45-59). Villatuerta: Txalaparta.

Foucault, Michel (1986). Of Other Spaces. Diacritics, 16(1), 22-27.

Halberstam, Jack (5 de julio, 2014). You Are Triggering me! The NeoLiberal Rhetoric of Harm, Danger and Trauma [Bully Bloggers]. Recuperado de: https://bullybloggers.wordpress.com/2014/o7/o5/you-are-triggeringme-the-neo-liberal-rhetoric-of-harm-danger-and-trauma/ [Consultado el 1 de noviembre de 2018].

Jones, Angela (2009). Queer Heterotopias: Homonormativity and the Future of Queerness. Interalia, a Journal of Queer Studies, 4, 1-20.

Kenny, Moira Reichel (2001). Mapping Gay L.A.: The Intersection of 
Place and Politics. Philadelphia: Temple University Press.

La Hoguera Transfeminista (2014). Qué queremos... [Hoguera]. Recuperado de: https://lahogueratransfeminista.wordpress.com/acerca-de/ [Consultado el 1 de noviembre de 2018].

Lefebvre, Henri (1974). La producción del espacio. Revista de Sociología, 3, 219-228.

Migrantes Transgresorxs (2017). Manada Antirracista Transfronteriza. Manifestación 28J. Orgullo Crítico [Migrantes Transgresorxs]. Recuperado de: http://migrantestransgresorxs.blogspot.pt $/ 2017 / 06 /$ manadaantirracista-transfronteriza.html [Consultado el 1 de noviembre de 2018].

Mouffe, Chantal (1993). The Return of the Political, Foreign Affairs. Londres y Nueva York: Verso.

Mouffe, Chantal (2016). Democratic Politics and Conflict: An Agonistic Approach. Política Común, 9, 1-6. doi: 10.3998/pc.12322227.0009.011.

Ochoa, Santi (2017). Manifestación Orgullo Crítico 2017. Kaos en la Red. Recuperado de: http://kaosenlared.net/manifestacion-orgullocritico-2017/. [Consultado el 26 de enero de 2019].

Orgullo Crítico (2017). OCM 2017 [Orgullo Crítico Madrid]. Recuperado de: https://orgullocritico.wordpress.com/2017/o4/24/ocm2017/ [Consultado el 1 de noviembre de 2018].

Orgullo Crítico (2018). Comunicado del Orgullo Crítico sobre los acontecimientos de la manifestación [Orgullo Crítico]. Recuperado de: https://www.facebook.com/orgullocriticomadrid/ [Consultado el 1 de noviembre de 2018].

Pérez Navarro, Pablo (2014). Queer Politics of Space in the 15-M Movement. Lambda Nordica, 19(2), 83-114.

Pérez Navarro, Pablo (2015). «Where Is My Tribe»? Queer Activism in the Occupy Movements. Interalia. A journal of queer studies. Recuperado de: http://www.interalia.org.pl/en/artykuly/on_a_rolling_basis/where_i s_my_tribe_queer_activism_in_the_occupy_movements.htm.

Preciado, Paul B. (2003). Multitudes Queer. Notes pour une politiques des 'anormaux'. Multitudes, 12(2), 17-25. doi: 10.3917/mult.012.0017.

Preciado, Paul B. (2010). Pornotopía. Arquitectura y sexualidad en la 'Playboy' durante la Guerra Fría. Madrid: Anagrama.

Preciado, Paul B. (2013). Decimos Revolución. En Solá, Miriam y Urko, 
Elena (Comp.). Transfeminismos. Epistemes, fricciones y flujos (915). Villatuerta: Txalaparta.

Puar, Jasbir (2017). Ensamblajes terroristas, el homonacionalismo en tiempos queer. Barcelona: Bellaterra.

Quinan, Christine (2016). Safe Space. En Rodríguez, Nelson M., Martino, Wayne J., Ingrey, Jennifer C. y Brockenbrough, Edward (Ed.). Critical Concepts in Queer Studies and Education (361-369). New York: Palgrave Macmillan.

Red PutaBolloNegraTransFeminista (2009). Manifiesto para la insurrección transfeminista [Destroying muros]. Recuperado de: http://ideadestroyingmuros.blogspot.pt/2009/12/manifiesto-para-lainsurreccion.html [Consultado el 1 de noviembre de 201].

Redondo, Mónica (26 de marzo, 2015). Topología Transmaricabollo, Píkara Magazine. Recuperado de: http://www.pikaramagazine.com/2015/o3/topologiatransmaricabollo/ [Consultado el 1 de noviembre de 2018].

Robles, Ricky G. (2018). Repensando los bloques no mixtos [Orgullos Críticos do Sull. Recuperado de: https:/orgulloscriticos.wordpress.com/2018/o3/o4/repensando-losbloques-no-mixtos/ [Consultado el 1 de noviembre de 2018].

Solá, Miriam (2013). Pre-textos, con-textos y textos. En Solá, Miriam y Urko, Elena (Comp.). Transfeminismos. Epistemes, fricciones y flujos (15-31). Villatuerta: Txalaparta.

Trujillo, Gracia (27 de junio, 2017). 40 Años Después. CTXT Contexto y Acción. Recuperado de: http://ctxt.es/es/20170621/Firmas/13514/ctxt-orgullo-critico-LGBTIqueer-trans.htm [Consultado el 1 de noviembre de 2018].

VV. AA. (2013). Transfeminismos. Epistemes, fricciones y flujos. Villatuerta: Txalaparta.

Weeks, Jeffrey (1995). Invented Moralities: Sexual Values in an Age of Uncertainty. Malden: Polity Press. 